\title{
EFECTO DE LA INUNDACIÓN SOBRE PARÁMETROS DE CRECIMIENTO DE STEVIA (Stevia rebaudiana Bertoni)
}

\section{EFFECT OF FLOODING ON GROWTH PARAMETERS OF STEVIA (Stevia rebaudiana Bertoni)}

\author{
Alfredo Jarma-Orozco ${ }^{1}$, Teobaldis Mercado-Fernández ${ }^{2}$, Jose Alejandro Cleves-Leguizamo ${ }^{3}$
}

\begin{abstract}
${ }^{1}$ Ingeniero Agrónomo, MSc. Fisiología de Cultivos, PhD Fisiología de Cultivos, Profesor Titular Facultad Ciencias Agrícolas. Universidad de Córdoba, carrera 6 No. 76-103, Montería, Colombia, e-mail: ajarma@correo.unicordoba.edu.co; ${ }^{2}$ Ingeniero Agrónomo, MSc. Hidrociencias, Ph.D. Hidrociencias, Profesor Titular Facultad Ciencias Agrícolas. Universidad de Córdoba, carrera 6 No. 76-103, Montería, Colombia, e-mail: teobaldis@correo.unicordoba.edu.co; ${ }^{3}$ Ingeniero Agrónomo, MSc. Sistemas de Producción Agrícola, (c) PhD. Agroecología, Profesor Asociado, Facultad Seccional Duitama, Escuela Administración de Empresas Agropecuarias. Universidad Pedagógica y Tecnológica de Colombia (UPTC), carrera 18 No. 22 00, Tunja, Colombia, e-mail: jose.cleves@uptc.edu.co
\end{abstract}

Rev. U.D.C.A Act. \& Div. Cient. 18(2): 393-399, Julio-Diciembre, 2015

\section{RESUMEN}

Stevia rebaudiana Bertoni es una planta de reciente introducción y cultivo en Colombia, donde se siembra en pequeñas extensiones, debido, entre otros aspectos, al desconocimiento de su sensibilidad a periodos prolongados de inundación. Esta situación, se registra con relativa frecuencia en algunos meses del año, en el Departamento de Córdoba. El presente estudio, se realizó en esta región, con el objetivo de evaluar los efectos de diferentes tiempos de inundación sobre el crecimiento de la especie. Para ello, se sometió el cultivo a inundación antes, durante y después de floración, durante 6, 12, 24, 48 y 72 horas. Los resultados indicaron que en todos los estados fisiológicos evaluados, las plantas mueren después de 24 horas de inundación, con significativas reducciones del área foliar (80\%) y número de hojas (30\%) en floración; lo mismo sucedió con la biomasa total por planta, la cual, se redujo (21\%). En post-floración -en esta fase fisiológica- el estrés, por hipoxia, indujo una distribución preferencial de biomasa hacia la raíz en detrimento de la del tallo. Los resultados de este trabajo indican que stevia es una planta altamente susceptible, a condiciones de estrés hídrico.

Palabras clave: Stevia rebaudiana, estrés hídrico, biomasa, área foliar, hipoxia.

\section{SUMMARY}

Stevia rebaudiana Bertoni, is a plant of recent introduction and cultivation in Colombia, where it is grown in small areas due, among other aspects, to the absence of the knowledge of its sensitivity to prolonged periods of flooding, common in the Department of Córdoba at certain times of the agricultural season. The present study was carried out in order to determine the effects of flooding on some growth variables of this species. The culture was subjected to flooding before, during and after flowering by periods of $6,12,24,48$ and 72 hours. The results indicated that in all evaluated physiological states, the plants died after 24 hours of flooding, with significant reductions in leaf area $(80 \%)$ and number of leaves (30\%) at blooming, as well as the total biomass per plant which was reduced by $21 \%$. At the post-flowering physiological stage, hypoxia stress induced a preferential distribution of biomass to the root at the expense of the stem. The results of this study indicate that stevia is a plant, highly susceptible to water stress conditions.

Key words: Stevia rebaudiana, hypoxia, stress, biomass, leaf area, stevia.

\section{INTRODUCCIÓN}

Ceunen et al. (2012) indican que Stevia rebaudiana Bertoni, conocida como ka'ahe'e o, simplemente, como stevia, es una especie que aún crece en estado silvestre, en terrenos arenosos y de buen drenaje en la región Oriental de Paraguay, lugar de origen y de dispersión.

Actualmente, tiene un gran reconocimiento por sus extraordinarias características organolépticas, debido a que posee una serie de moléculas endulzantes no calóricas, 300 veces más dulce que el azúcar común. Es muy utilizada en China, en Estados Unidos, en Japón, en Corea, en Australia, 
en Nueva Zelanda y en muchos países de la Unión Europea (Hitesh et al. 2013).

Los cambios fisiológicos más notables en las plantas están relacionados con las variaciones significativas con los factores climáticos, como radiación, precipitación, temperatura, contenidos de humedad en el suelo y presión de vapor. Estas variaciones inciden en el crecimiento y en el desarrollo de la especie vegetal, manifestadas a través de cambios bioquímicos, como tasas de fotosíntesis, transpiración, síntesis de moléculas, además de variaciones morfológicas, como la modificación del área foliar y la distribución de la biomasa, entre otros (Jarma, 2008). Algunos autores consideran que estas estrategias de adaptación se presentan de manera proporcional a los gradientes hidrológicos de estrés, como la cantidad y el tiempo de inundación (Magee \& Kentula, 2005).

La inundación es una situación de exceso de agua en la zona radicular. La disminución del oxígeno en el suelo, debido al exceso de agua, resulta en hipoxia o anoxia, responsables de las mayores pérdidas en la productividad de los cultivos (Jiménez et al. 2012). En ese sentido, las respuestas de las plantas está determinada por la habilidad que pueda tener la raíz para adquirir oxígeno y el desarrollo del área foliar específica.

Mielke et al. (2005) indican que muchas especies, bajo condiciones de anoxia, pueden cambiar los patrones de asignación de biomasa en la planta y que pueden ocurrir aumentos proporcionales en la biomasa de la raíz, para formar aerénquima, a expensas del crecimiento total, ya que la biomasa del tejido fotosintético disminuye, como también lo hace la ganancia de carbono fotosintético, probablemente, por limitaciones estomáticas (Herrera et al. 2008).

Kissmann et al. (2014) refieren que los cambios pueden ser lentos o rápidos, dependiendo de la especie. Otros autores afirman que, generalmente, los cambios bioquímicos están asociados a períodos cortos de estrés por anoxia, en tanto que los cambios morfológicos, están relacionados con períodos más largos de inundación (Jiménez et al. 2012).

Las respuestas morfo-fisiológicas diferenciales entre especies sometidas a estrés por inundación han sido documentadas por Hough-Sneea et al. (2014), quienes reportaron que, bajo estas condiciones, la planta acumula menos biomasa total y re-asigna la restante, destinando una mayor proporción a la raíz y reduciendo el área foliar total y el área foliar específica.

Por su parte, Kissmann et al. (2014) indican que la reducción en la expansión del área foliar trae consecuencias drásticas en la producción de biomasa y en el crecimiento de la planta; sin embargo, otros autores indican que la planta reduce el crecimiento de la raíz, ya sea por la presencia de iones fitotóxicos en el suelo, tales como $\mathrm{Mn}^{2+}, \mathrm{Fe}^{2+}$ y S ${ }^{2-}$ (Yamauchi et al. 2013) o porque sólo puede crecer cerca de la superficie del suelo, procurando el poco oxígeno disponible (Corrêa de Souza et al. 2013; Baracaldo et al. 2014).

De igual manera, la falta de oxígeno -derivada de las condiciones de inundación- disminuye la respiración. La producción de energía es restringida a fermentación, la que deriva solamente dos ATPs; adicionalmente, la hipoxia causa reducciones en la conductividad hidráulica y afecta las aquaporinas, disminuyendo la absorción de agua y nutrientes por la raíz (Horchani et al. 2008). Asimismo, cuando se producen especies reactivas de oxígeno (EROs), que pueden causar daños al metabolismo de la célula por oxidación de lípidos y de proteínas, la planta induce mecanismos enzimáticos y no enzimáticos, para controlar esas EROs y proteger sus células (Karuppanapandian et al. 2011).

En este orden de ideas, Wood et al. (2000) manifiestan que en América Latina, el 11,3\% de las tierras cultivables presentan problemas de mal drenaje, principalmente, por la fisiografía. En los suelos del Valle del río Sinú, Córdoba, Colombia, en algunas épocas del año, por lo general, entre agosto y noviembre, se presentan altas precipitaciones, asociadas a deficientes drenajes, asociados a topografías llanas, niveles freáticos altos y el predominio de texturas franco-arcillosas.

Algunos estudios sobre stevia en la región indican que los suelos, para su producción, deben ser preferiblemente de topografía plana, pero no inundables (Jarma, 2008). Sobre la base de las ideas expuestas, la presente investigación tuvo por objetivo estudiar algunas respuestas morfológicas de stevia, sometida a diferentes tiempos de inundación, bajo las condiciones ambientales del Valle del Sinú.

\section{MATERIALES Y MÉTODOS}

La investigación, se realizó entre abril y agosto de 2012, en la Granja Experimental de la Facultad de Ciencias Agrícolas de la Universidad de Córdoba, con coordenadas geográficas $8^{\circ} 44^{\prime} 52^{\prime \prime} \mathrm{N}$ y $75^{\circ} 52^{\prime} 53^{\prime \prime} \mathrm{W}$. La zona posee -en promedio anual- una temperatura de $28^{\circ} \mathrm{C}$, humedad relativa del $84 \%$, insolación entre 1400 y 1800 horas luz/año, precipitación de $1200 \mathrm{~mm}$ y una altitud de $15 \mathrm{msnm}$ (Palencia et al. 2006).

Los estudios, se hicieron en pre-floración, floración y postfloración en cada uno, de los cuales, se estableció un diseño completamente aleatorizado, con 6 tiempos de inundación: $6,12,24,48$ y 72 horas y un testigo absoluto, que se mantuvo a capacidad de campo sin períodos de inmersión y cuatro repeticiones. Cada unidad experimental estuvo constituida por diez plantas, para un total de 720 . Cada planta fue establecida en bolsa de polietileno de 22 x $35 \mathrm{~cm}$ calibre 3 , 
con sustrato en relación 1:1:1 (cascarilla de arroz, aluvión y arena), previamente desinfectado. Las variables respuesta, se tomaron diez días después de haber retirado las plantas de cada tratamiento de inmersión.

Las respuestas, se cuantificaron, a través del área foliar (AF), medida en $\mathrm{cm}^{2}$, con el software Determinador Digital de Áreas (DDA); el número total de hojas por planta (NH) y la masa seca $(\mathrm{g})$ asignada a la raíz (MSR); el dosel (MSD) y la total $\left(M S_{T}\right)$, determinada al someter los tejidos a $72^{\circ} \mathrm{C}$, durante 48 a 72 horas. Los datos fueron procesados y analizados por el programa SAS versión 9,2 y las gráficas, se ilustraron con el programa Sigma Plot versión 10,0.

\section{RESULTADOS Y DISCUSIÓN}

Debido a la muerte de la las plantas en los períodos de inundación superiores a 24 horas, sólo fue posible tomar información para períodos de inundación de 6, 12 y 24 horas. Los resultados obtenidos son consistentes con otras especies, como en el caso de Benz et al. (2007), quienes encontraron que la hipoxia ocasionaba deterioro de las raíces y muerte de las plantas del complejo Piriqueta caroliniana; asimismo, Martínez-Alcántara et al. (2012) coinciden que la hipoxia causa inhibición del crecimiento y puede conllevar a mortalidad total de las plantas, por los efectos sobre el metabolismo del vegetal, revisados en el acápite anterior.

Área foliar por planta (AF): En cualquiera de los tres estados de desarrollo, se presentó un efecto negativo sobre el AF $(\mathrm{P} \leq 0.01)$, a partir de seis horas de inundación, tal como se indica en la tabla 1; probablemente, las plantas sometidas a estrés por inundación enviaron señales tempranas desde la raíz hasta el dosel, para inducir el cierre estomático y, de esta forma, el intercambio gaseoso es interrumpido y las tasas de producción de biomasa tienden a disminuir. Al afectarse la asimilación de carbono -por causa de la hipoxia-, la reducción en la expansión del área foliar representa para la planta una estrategia para ahorrar energía, posiblemente, reduciendo la respiración para mantener su metabolismo tan bajo, como sea posible.

La reducción del AF bajo condiciones de hipoxia por inundación ha sido documentadas en fique, Furcraea castilla y F. macrophylla (Casierra \& Gómez, 2008); en fresa, Fragaria sp. (Casierra \& Vargas, 2007); en aguacate, Persea americana (Besoain et al. 2005); en sorgo, Sorghum bicolor (Promkhambut et al. 2010); en maíz, Zea mays (Odiyi, 2013) y en otras especies (Mielke \& Schaffer, 2010). La inundación afecta adversamente la formación y la expansión de las hojas, por lo que se induce senescencia prematura de estas, lo cual, se traduce en una inhibición en el crecimiento del dosel, debido, quizás, a reducciones en la extensibilidad de las paredes celulares o por acumulación de sustancias tóxicas en las hojas, como el etanol.

Número de hojas por planta (NH): Plantas inundadas por seis horas o más mostraron una reducción significativa $(p<0.05)$ en el $\mathrm{NH}$ en floración y en post-floración (Tabla 1). La abscisión, probablemente, fue causada por una senescencia prematura de las mismas o una baja tasa de iniciación de foliolos, tal como lo proponen Stewart et al. (2010), en Populus angustifolia y Kissmann et al. (2014), en Styrax pohlii; estos últimos autores encontraron reducción del número de hojas y del área foliar después 100 días de inundadas las plantas.

Casierra \& Vargas (2007) señalan que la abscisión y la senescencia prematura de hojas, causadas por la inundación, se fundamenta en desórdenes hormonales y restricción en la disponibilidad de ATP y agua. Aunque las hormonas no fueron cuantificadas es probable que la reducción en el $\mathrm{NH}$ de plantas de stevia inundadas sea una consecuencia de un desbalance hormonal, que estaría dado, principalmente, por una reducción en la síntesis de giberelinas y citoquininas, como lo propone López (2009) y aumentos en la concen-

Tabla 1. Área foliar y número de hojas de Stevia rebaudiana Bertoni, sometida a diferentes períodos de inundación, en tres estados fenológicos de desarrollo (Pre-fl=prefloración; Flor=floración; Post-fl=postfloracion).

\begin{tabular}{|c|c|c|c|c|c|c|}
\hline \multirow{2}{*}{$\begin{array}{l}\text { Inundación } \\
\text { (h) }\end{array}$} & \multicolumn{3}{|c|}{ Área foliar/planta $\left(\mathrm{cm}^{2}\right)$} & \multicolumn{3}{|c|}{ Número de hojas/planta } \\
\hline & Pre-fl & Flor & Post-fl & Pre-fl & Flor & Post-fl \\
\hline 0 & 256,3 a & 293,0 a & $145,0 \mathrm{a}$ & $155,7 \mathrm{a}$ & $158,2 \mathrm{a}$ & $185,3 \mathrm{a}$ \\
\hline 6 & $198,5 \mathrm{~b}$ & $157,7 \mathrm{~b}$ & $133,0 \mathrm{~b}$ & 152,8 a & $113,2 \mathrm{~b}$ & $155,3 \mathrm{ab}$ \\
\hline 12 & $135,1 \mathrm{~b}$ & $134,3 \mathrm{~b}$ & $124,0 \mathrm{~b}$ & $151,1 \mathrm{a}$ & $111,7 \mathrm{~b}$ & $127,5 \mathrm{~b}$ \\
\hline 24 & $115,6 \mathrm{c}$ & $57,8 \mathrm{c}$ & $77,3 \mathrm{c}$ & 136,5 a & $109 \mathrm{~b}$ & $132,8 b$ \\
\hline C.V. (\%) & 20,8 & 15,5 & 15,8 & 15,0 & 22,8 & 15,9 \\
\hline
\end{tabular}

Promedios con la misma letra en una columna son iguales estadísticamente, de acuerdo al test protegido de Tukey $(0,05)$. 
tración de ácido abscísico (ABA), auxinas y etileno. Está documentado que, bajo condiciones de hipoxia, en la raíz se favorece la síntesis de SAM (S-Adenilato-Metionina) y su paso a ACC (Ácido aminociclopropano-1-carboxílico); que al ascender vía xilema, en presencia de oxígeno, se transforma en etileno, hormona que, junto con el ABA, causan abscisión de estructuras en el dosel.

Masa seca total por planta $\left(\mathrm{MS}_{\mathrm{T}}\right)$ : No se detectaron diferencias estadísticas significativas $(\mathrm{p}>0.05)$ de la $\mathrm{MS}_{\text {т }}$ por planta entre plantas inundadas y no inundadas en pre-floración y floración (2,5 y 4,2g, respectivamente); sin embargo, contrariamente a lo advertido en los dos primeros estados, en post-floración, se observó un efecto significativo $(p<0.05)$, que indicó una reducción del $21 \%$ de la $M \mathrm{MS}_{\mathrm{T}}$ de las plantas inundadas, respecto al control. Es importante acotar que la reducción de la MSt estaría explicada por los descensos en la masa seca del dosel $(44,0 \%)$ y no de la raíz, en la que se percibió un aumento de un $39 \%$ al someterse a estrés por inundación (Tabla 2).

Kissmann et al. (2014) reportaron que las condiciones de inundación en Styrax pohlii antes de 50 días no causaron efecto sobre el número de hojas, el área foliar y la biomasa total de la planta, aunque estas diferencias sí fueron evidentes después de 100 días de inundación; es probable, que la condición observada en stevia para la $M S_{\text {, }}$, no varíe posteriormente, como lo proponen los autores para S. pohlii, ya que, como se mencionó, los porcentajes de mortalidad fueron tan altos, que indican la alta susceptibilidad de la especie a la inundación después de 24 horas.

Stevia es una especie semi-perenne, cuya raíz es pivotante, filiforme y no profundiza, distribuyéndose cerca de la superficie. A diferencia de la stevia, que se comportaría como especie susceptible, muchos árboles tropicales son tolerantes a suelos inundables, en los que se incluyen Tabebuia avellanedae (Davanso et al. 2002) y Erythrina speciosa; probablemente en estas especies, se realiza una baja actividad metabólica, bajo condiciones de escasez de oxígeno en el suelo, como consecuencia de una baja tasa de producción de energía, al restringirse la síntesis de ATP (Medina et al. 2009).

Relación masa seca raíz / masa seca dosel $\left(M_{\mathrm{R}} / \mathrm{MS}_{\mathrm{D}}\right)$ : En pre-floración y en floración la relación $M S_{R} / M S_{D}$ no mostró variación estadística significativa entre plantas inundadas y no inundadas ( $p>0.05)$, aunque este efecto sí fue evidente en post-floración (Figura 1). Esta relación acusó valores bajos en las plantas no estresadas $(0,42 \pm 0,03 E E ; n=10)$, en tanto, que en cualquiera de los niveles de estrés por inundación, la relación se mantuvo alta y constante $(1,52$ $\pm 0,11 \mathrm{EE} ; \mathrm{n}=10$ ), lo que sugiere que en stevia existe una re-distribución de biomasa entre la raíz y el dosel, a partir de seis horas de inundación.

Las plantas inundadas ubicaron mayor biomasa en la raíz, como mecanismo de defensa al prolongar su sistema de exploración en busca de oxígeno, formando, rápidamente, raíces adventicias u otros órganos y este rápido crecimiento, se efectúa a expensas de los asimilados, que deberían ir normalmente al dosel, donde se observaron decrecimientos, bajo condiciones de inundación (Tabla 2).

Resultados contrastantes en tomate han sido reportados por Baracaldo et al. (2014), quienes informaron que, bajo condiciones de anoxia, las plantas redujeron significativamente la biomasa de la raíz, a partir de ocho días de inundación; por su parte, Casierra \& Vargas (2007), en estudios con tres genotipos de fresa, encontraron

Tabla 2. Masa seca de raíz, dosel (hojas + tallo) y total de Stevia rebaudiana Bertoni, sometida a diferentes períodos de inundación, en post-floración. Medias \pm Error Estándar, n=10.

\begin{tabular}{|c|c|c|c|}
\hline \multirow{2}{*}{ Inundación (h) } & \multicolumn{3}{|c|}{ Masa Seca $(\mathrm{g})$} \\
\hline & Raíz $\left(\mathrm{MS}_{\mathrm{R}}\right)$ & Dosel (MSD) & Total $\left(\mathrm{MS}_{\mathrm{T}}\right)$ \\
\hline 0 & $1,52 \pm 0,12 a$ & $3,62 \pm 0,30 a$ & $5,14 \pm 0,40 a$ \\
\hline 6 & $2,70 \pm 0,20 b$ & $1,75 \pm 0,27 b$ & $4,45 \pm 0,44 b$ \\
\hline 12 & $2,26 \pm 0,24 b$ & $1,49 \pm 0,07 b$ & $3,75 \pm 0,31 b$ \\
\hline 24 & $2,40 \pm 0,27 b$ & $1,62 \pm 0,25 b$ & $4,02 \pm 0,52 b$ \\
\hline C.V. (\%) & 21,3 & 13,2 & 14,7 \\
\hline
\end{tabular}

Promedios con la misma letra en una columna son iguales estadísticamente, de acuerdo al test protegido de Tukey $(0,05)$. 


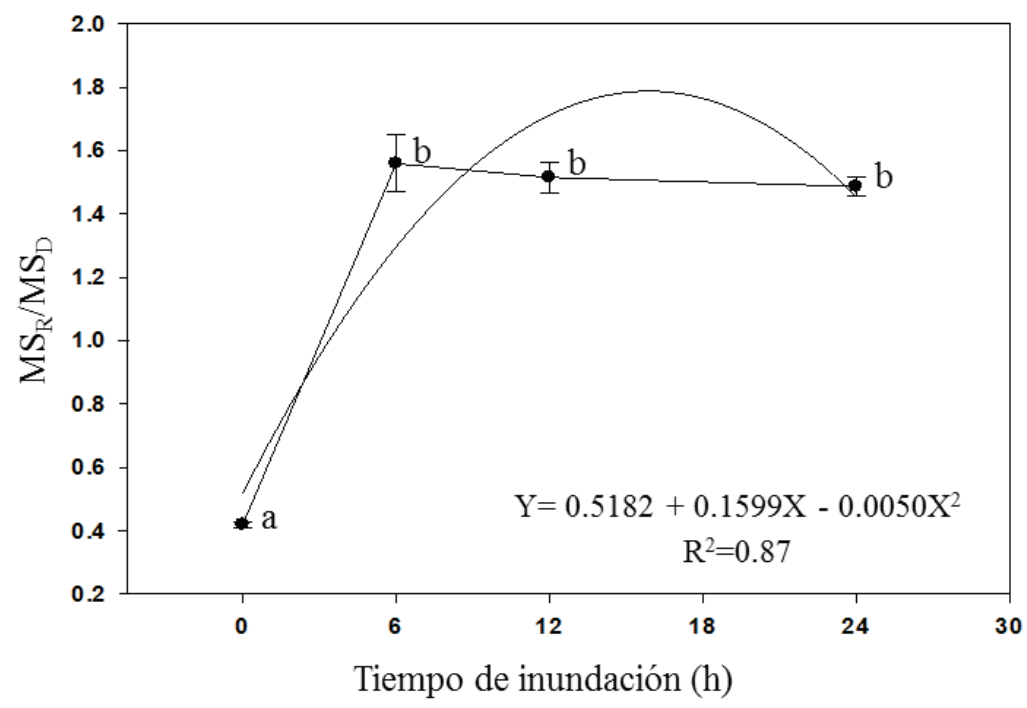

Figura 1. Relación masa seca de raíz y masa seca del dosel (MSR/MSD) de Stevia rebaudiana Bertoni, sometida a diferentes períodos de inundación, en post-floración. Promedios con la misma letra son iguales estadísticamente, de acuerdo al test protegido de Tukey $(0,05)$.

aumentos en la biomasa de raíz, aunque no en el total de la planta, argumentando que esta condición fue debida a la disminución del crecimiento de órganos aéreos y emisión de raíces adventicias en el cuello del raíz.

Diversos autores han reportado una re-distribución de biomasa en especies susceptibles a encharcamiento, creciente hacia las raíces y decreciente hacia el dosel (Hough-Sneea et al. 2014; Kissmann et al. 2014); lo anterior, generalmente, es debido a la formación de nuevas raíces adventicias, lenticelas y espacios intercelulares estrechos, así como el efecto negativo sobre el crecimiento de la parte aérea, mediante la inhibición de la formación de nuevas hojas, la expansión foliar y el crecimiento de entrenudos (Baracaldo et al. 2014). Esto es una consecuencia de la privación de $\mathrm{O}_{2}$, que afecta las tasas de intercambio de gases, las relaciones hídricas, el equilibrio nutricional y hormonal y la partición del carbono, con efectos marcados sobre el crecimiento y la supervivencia de muchas especies.

Atkinson et al. (2008) indicaron que, bajo inundación, los productos de la ruta fermentativa acetaldehído y etanol, pueden ser volatilizados, a través de lenticelas o transportados a las hojas vía corriente transpiratoria; con estas condiciones, el etanol en las hojas puede ser convertido a Acetil-CoA y usado en el metabolismo del carbono. En ese sentido, Mielke \& Schaffer, (2010), trabajando con Eugenia uniflora L., a pesar de no haber observado lenticelas en las plantas inundadas, sugieren que los bajos rendimientos de biomasa del dosel y la alta relación $M S_{R} / M S_{D}$, se pudieron deber a una excesiva acumulación de acetaldehído y de etanol, como una consecuencia del desbalance, en dicha relación, asociada con la baja capacidad del dosel, para liberar estos compuestos tóxicos.

Corrêa de Souza et al. (2013), en trabajo con plantas inundadas y no inundadas de Garcinia brasiliensis (Mart.), encontraron reducciones significativas en la masa seca de raíz (36\%), de hojas (44\%) y de dosel (35\%), aunque no detectaron diferencias en la masa seca del tallo ni en la relación masa seca de raíz/dosel, evidenciando que esta relación se mantuvo a expensas de crecimientos diferenciados de los órganos involucrados. Lo anterior, es contrario a lo observado en la presente investigación y confirma la diversidad de respuestas entre especies ante condiciones de hipoxia, tal como lo reportan Hernández et al. (2010). Estos autores informaron que los resultados en $G$. brasiliensis pueden estar explicados por la menor cantidad de raíces de diámetro fino y de longitud larga, que son altamente sensibles a la hipoxia y a la presencia de lenticelas hiper-atrofiadas en la base del tallo que, probablemente, contribuyeron a la ventilación de las raíces.

Del presente estudio, se concluye que, bajo condiciones de inundación entre 6 y 24 horas, se observa una reducción del área foliar de las plantas de stevia, en cualquier estado 
fenológico, debido a una pérdida significativa en su número de hojas, probablemente, inducidos por cambios hormonales y bioquímicos, que derivan en marchitamiento, en necrosis, en senescencia prematura y en abscisión. Por otra parte, en post-floración, la masa seca de las raíces aumenta en condiciones de inundación, al parecer, a expensas de la biomasa del dosel que decrece, lo que demuestra una rápida re-distribución de asimilados, como mecanismo espaciotemporal de defensa.

Además, se puede inferir que Stevia rebaudiana registra altos índices de mortalidad después de 24 horas de inundación, lo que la ubicaría en el grupo de especies altamente susceptibles, a condiciones de hipoxia en el suelo; sin embargo, la especie podría presentar algunos mecanismos de defensa temprana (24 horas), los cuales, se hacen más evidentes en estado de post-floración, aunque se debe demostrar. Con base en ello, los cultivadores de esta especie deben tomar medidas de precaución o corrección, para evitar que el cultivo permanezca por más de 6 horas en condiciones de inundación, por lo que se deben realizar drenajes inmediatos, antes de este tiempo.

Agradecimientos: Los autores agradecen a los Ingenieros Agrónomos William A. Romero, Francisco Peña, Dairo Pérez y José Villalba y a los auxiliares del Laboratorio de Fisiología Vegetal de la Facultad de Ciencias Agrícolas de la Universidad de Córdoba. Conflicto de intereses: El manuscrito fue preparado y revisado con la participación de todos los autores, quienes declaramos que no existe conflicto de intereses que ponga en riesgo la validez de los resultados presentados.

\section{BIBLIOGRAFÍA}

1. ATKINSON, C.; HARRISON-MURRAY, R.; TAYLOR, J. 2008. Rapid flood-induced stomatal closure accompanies xylem sap transportation of rootderived acetaldehyde and ethanol in Forsythia. Environ. Exp. Bot. 64:196-205.

2. BARACALDO, A.; CARVAJAL, R.; ROMERO, P.; PRIETO, A.; GARCÍA, F.; FISCHER, G.; MIRANDA, D. 2014. El anegamiento afecta el crecimiento y producción de biomasa en tomate chonto (Solanum lycopersicum L.), cultivado bajo sombrío. Rev. Col. Cienc. Hort. 8(1):91-102.

3. BENZ, B.; RHODE, J.; CRUZAN, M. 2007. Aerenchyma development and elevated alcohol dehydrogenase activity as alternative responses to Hypoxic soils in the Piriqueta caroliniana complex. Am. J. Bot. 94(4):542-550.
4. BESOAIN, X.; ARENAS, C.; SALGADO, E.; LATORRE, B. 2005. Effect of soil flooding period on the development of avocado (Persea americana) root rot caused by Phytophthora cinnamomi. Cien. Inv. Agr. 32(2):79-84.

5. CASIERRA-POSADA, F.; GÓMEZ, N. 2008. Crecimiento foliar y radical en plantas de fique (Furcraea castilla y $F$. macrophylla) bajo estrés por encharcamiento. Agr. Col. 26(3):381-388.

6. CASIERRA-POSADA, F.; VARGAS, Y. 2007. Crecimiento y producción de fruta en cultivares de fresa (Fragaria sp.) afectados por encharcamiento. Rev. Colomb. Cienc. Hort. 1(1):21-32.

7. CEUNEN, S.; WERBROUCK, S.; GEUNS, J. 2012. Stimulation of steviol glycoside accumulation in Stevia rebaudiana by red LED Light. J. Plant Physiol. 169:749-752.

8. CORRÊA DE SOUZA, T.; SOUZA, E.; DOUSSEAU, S.; DE CASTRO E.; MAGALHÃES, P. 2013. Seedlings of Garcinia brasiliensis (Clusiaceae) subjected to root flooding: Physiological, morpho-anatomical, and antioxidant responses to the stress. Aquatic Botany 111:43-49.

9. DAVANSO, V.; SOUZA, L.; MEDRI, M.; PIMENTA, J.; BIANCHINI, E. 2002. Photosynthesis, growth and development of Tabebuia avellanedae Lor. exGriseb. (Bignoniaceae) in flooded soil. Braz. Arch. Biol. Technol. 45:375-384.

10. HERNÁNDEZ, R.; RADA, F.; SILVA, R. 2010. Crecimiento y desarrollo en plantas juveniles de apamate (Tabebuia rosea (Bertol.) A. Dc.) sometidas a inundación. Pittieria. 34:113-126.

11. HERRERA, A.; TEZARA, W.; MARIN, O.; RENGIFO, E. 2008. Stomatal and non-stomata limitations of photosynthesis in trees of a tropical seasonally flooded forest. Physiol. Plant. 134:41-48.

12. HITESH, K.; KIRAN, K.; SUPHLA, B.; VIJAY, K.; SANJAY, K. 2013. A comprehensive analysis of fifteen genes of steviol glycosides biosynthesis pathway in Stevia rebaudiana (Bertoni). Gene. 492:276-284.

13. HORCHANI, F.; ALOUI, A.; BROUQUISSE, R.; ASCHISMITI, S. 2008. Physiological responses of tomato plants (Solanum lycopersicum) as affected by root hypoxia. J. Agron. Crop Sci.197:297-303. 
14. HOUGH-SNEEA, N.; NACKLEYA, L.L.; KIMA, S.; EWINGA, K. 2014. Does plant performance under stress explain divergent life history strategies? The effects of flooding and nutrient stress on two wetland sedges. Aquat. Bot. (2014) Disponible desde Internet en: http://dx.doi.org/10.1016/j.aquabot.2014.03.001 (con acceso 17/02/2015).

15. JARMA-OROZCO, A. 2008. Estudios de adaptación y manejo integrado de estevia (Stevia rebaudiana Bert.): nueva alternativa agroindustrial del Caribe colombiano. Una revisión. Rev. Col. Sc. Hort. 2(1):109-120.

16. JIMÉNEZ, J.; MORENO, L.; MAGNITSKIY, S. 2012. Respuesta de las plantas a estrés por inundación. Una revisión. Rev. Col. Cienc. Hort. 6(1):96-109.

17. KARUPPANAPANDIAN, T.; MOON, J.; KIM, C.; MANOHARAN, K.; KIM, W. 2011. Reactive oxygen species in plants: their generation, signal transduction, and scavenging mechanisms. Aust. J. Crop Sci. 5:709-725.

18. KISSMANN, C.; BORGES, E.; TERUYA, M.; HABERMANN, G. 2014. Morphological effects of flooding on Styrax pohlii and the dynamics of physiological responses during flooding and post-flooding conditions. Aquatic Botany. 119:7-14.

19. LÓPEZ, O. 2009. Fisiología y ecología de comunidades arbóreas en hábitats inundables. Acta Biol. Panam. 1:68-86.

20. MAGEE, T.; KENTULA, M. 2005. Response of wetland plant species to hydrologic conditions. Wetlands Ecol. Managem. 13:163-181.

21. MARTÍNEZ-ALCÁNTARA, B.; JOVER, S.; QUIÑONES, A.; FORNER-GINER, M.; RODRÍGUEZ-GAMIR, J.; LEGAZ, F.; PRIMO-MILLO, E.; IGLESIAS, D. 2012. Flooding affects uptake and distribution of carbon and nitrogen in citrus seedlings. J. Plant Physiol. 169:1150-1157.

22. MEDINA, C.; SANCHES, M.; TUCCI, M.; SOUSA, C.; CUZZUOL, G.; JOLY, C. 2009. Erythrina speciosa (Leguminosae-Papilionoideae) under soil water saturation: morphophysiological and growth responses. Ann. Bot. 104:671-680.

23. MIELKE, M.; MATOS, E.; COUTO, V.; DE ALMEIDA, A.; GOMES, F.; OLIVEIRA, M. 2005. Algumas respostas fotossintéticas e do crescimento de plántulas de Annona glabra L. ao alagamento do solo. Acta Bot. Bras. 19(4):905-911.

24. MIELKE, M.; SCHAFFER, B. 2010. Effects of soil flooding and changes in light intensity on photosynthesis of Eugenia uniflora L. seedlings. Acta Physiol Plant (2011) 33:1661-1668.

25. ODIYI, B. 2013. The effects of flooding and drought stress on the growth of maize (Zea maysL.) seedlings. J. Biol.FoodSci. Res. 2(3):30-32.

26. PALENCIA, G.; MERCADO, T.; COMBATT, E. 2006. Estudio Agroclimático del Departamento de Córdoba. Universidad de Córdoba, Colombia. 126p.

27. PROMKHAMBUT, A.; YOUNGER, A.; POLTHANEE, A.; AKKASAENG, C. 2010. Morphological and physiological responses of sorghum (Sorghum bicolor L. Moench) to waterlogging. Asian J. Plant Sci. 9(4):183-193.

28. STEWART, R.; NIELSEN, J.; SHENTON, L.; KM, G.; LETTS, M. 2010. Effects of flooding on leaf development, transpiration, and photosynthesis in narrow leaf cottonwood, a willow-like poplar. Photosynth. Res. 104:31-39.

29. WOOD, S.; SEBASTIAN, K.; SCHERR, S. 2000. Soil resource condition. En: Wood, S.; Sebastian, K.; Scherr, S (Eds).Pilot analysis of global ecosystems: Agroecosystems. International Food Policy Research Institute and World Resources Institute.Washington D.C. p.45-54.

30. YAMAUCHI, T.; SHIMAMURA, S.; NAKAZONO, M.; MOCHIZUKI, T. 2013. Aerenchyma formation in crop species: A review. Field Crops Res. 152:8-16.

Recibido: Abril 1 de 2015

Aceptado: Octubre 16 de 2015

\section{Cómo citar:}

Jarma-Orozco, A.; Mercado-Fernández, T., Cleves-Leguizamo, J.A. 2015. Efecto de la inundación sobre parámetros de crecimiento de stevia (Stevia rebaudiana Bertoni). Rev. U.D.C.A Act. \& Div. Cient. 18(2): 393-399. 